International Journal on Computational Science \& Applications (IJCSA) Vol.5, No.4,August 2015

\title{
AN APPROACH TO DECREASE DIMENTIONS OF LOG- ICAL ELEMENTS BASED ON BIPOLAR TRANSISTOR
}

\author{
E.L.Pankratov ${ }^{1,3}$ and E.A.Bulaeva ${ }^{1,2}$ \\ ${ }^{1}$ Nizhny Novgorod State University,23 Gagarin avenue, Nizhny Novgorod,603950,Russia \\ ${ }^{2}$ Nizhny Novgorod State University of Architecture and Civil Engineering, 65 Il'insky \\ street, Nizhny Novgorod, 603950,Russia \\ ${ }^{3}$ Nizhny Novgorod Academy of the Ministry of Internal Affairs of Russia, 3 Ankudinovs- \\ koe Shosse, Nizhny Novgorod, 603950, Russia
}

\begin{abstract}
In this paper we consider manufacturing logical elements with function AND-NOT based on bipolar transistors. Based on recently considered approach to decrease dimensions of solid state electronic devices with the same time increasing of their performance we introduce an approach to decrease dimensions of transistors and p-n-junctions, which became a part of the logical element. Framework the approach a heterostructure with required configuration should be manufactured. After the manufacture required areas of the heterostructures should be doped by diffusion or ion implantation. The doping should be finished by optimized annealing of dopant and/or radiation defects.
\end{abstract}

\section{KEYWORDS}

Transistor-transistor logic; optimization of manufacturing; decreasing of dimensions of transistor; analytical approach for modelling

\section{INTRODUCTION}

In the present time solid state electronic is intensively elaborating. Framework the elaborating one can find increasing of integrating rate of elements of integrated circuits ( $p-n$-junctions, their systems et al) with decreasing of their dimensions [1-7]. To ways of decreasing of dimensions of elements of integrated circuits are using laser and microwave types of annealing of dopants and/or radiation defects [8-16]. Another way to decrease the dimensions is using inhomogeneity of doped by diffusion or ion implantation heterostructure $[1-3,17]$. Using the second way leads to necessity to optimize annealing of dopants and/or radiation defects [17]. It is known, that radiation processing of materials leads to changing of distributions of concentrations of dopants [18]. In this situation radiation processing attracted an interest to decrease of dimensions of elements of integrated circuits $[17,18]$.

In the next paragraph we introduce an analytical approach to analyze mass transport during technological process. The approach gives a possibility to generalize recently considered in literature several limiting cases. Framework the approach we consider mass transport in a multilayer structures without crosslinking solutions on the interfaces between the layers. The approach also gives a possibility to optimize technological processes.

DOI:10.5121/ijcsa.2015.5401 


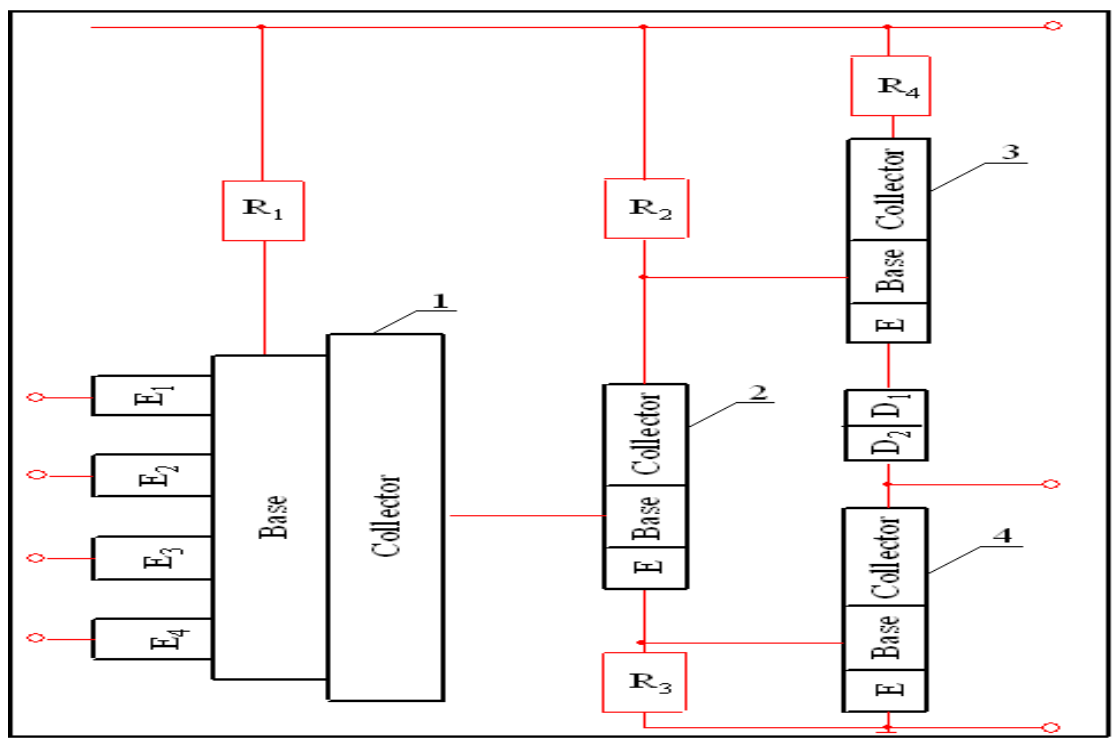

Fig. 1a. Structure of logical element based on bipolar transistor. View from top. Black marked transistors and $p$ - $n$-junction manufactured by using doping of appropriate sections of the epitaxial layer. Dimensions of these devices are decreased. Transistor 1 is a multiemitter transistor. Emitters have been marked by using letter E. The index indicates their number in the multiemitter transistor. $D_{1}$ and $D_{2}$ mean dopants of $p$ and $n$

types in $p$ - $n$-junction. Red marked resistors $\left(R_{i}\right)$ and wires have no decreasing of their dimensions

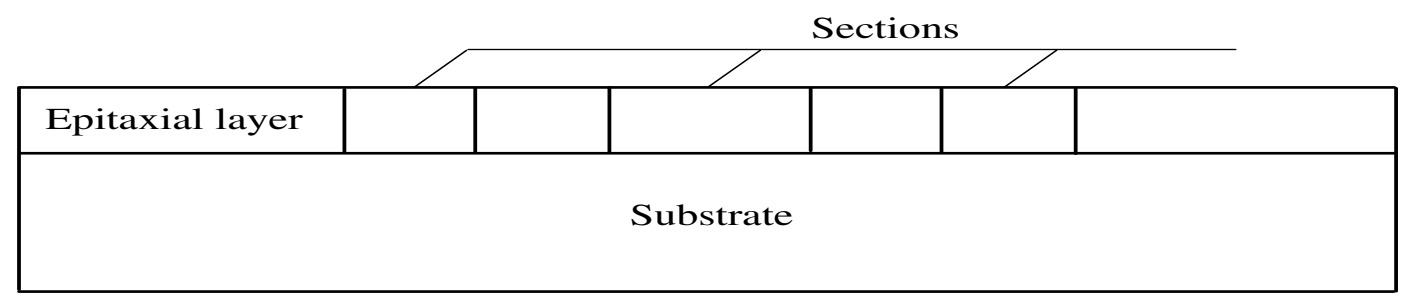

Fig. $1 b$. Heterostructure with a substrate and an epitaxial layer. Some sections have been manufactured by using another materials in the epitaxial layer

\section{METHOD OF SOLUTION}

In this paper we consider a heterostructure with a substrate and an epitaxial layer (see Figs. 1). Epitaxial layer includes into itself several sections manufactured by using another materials (see Figs. 1). The sections have been doped by diffusion or by ion implantation to generate required type of conductivity ( $p$ or $n$ ) during manufacturing transistors and $p$ - $n$-junction. After that annealing of dopant and/or radiation defects should be considered. Main aim of our paper is determination of conditions, which correspond to decreasing of the considered elements and increasing switching time of $p$ - $n$-junctions by analysis of redistribution of dopant and radiation defects during their annealing.

Let us determine spatio-temporal distributions of concentrations of dopants to solve our aim. We assume, that main part of transport of dopant during annealing is diffusion. Interaction between atoms of dopant and defects is small. In this situation we determine spatio-temporal distribution of concentration of dopant as solution the second Fick's law in the following form $[1,3,18]$ 


$$
\frac{\partial C(x, y, z, t)}{\partial t}=\frac{\partial}{\partial x}\left[D_{C} \frac{\partial C(x, y, z, t)}{\partial x}\right]+\frac{\partial}{\partial y}\left[D_{C} \frac{\partial C(x, y, z, t)}{\partial y}\right]+\frac{\partial}{\partial z}\left[D_{C} \frac{\partial C(x, y, z, t)}{\partial z}\right]
$$

Boundary and initial conditions for the equations are

$$
\begin{gathered}
\left.\frac{\partial C(x, y, z, t)}{\partial x}\right|_{x=0}=0,\left.\frac{\partial C(x, y, z, t)}{\partial x}\right|_{x=L_{x}}=0,\left.\frac{\partial C(x, y, z, t)}{\partial y}\right|_{y=0}=0,\left.\frac{\partial C(x, y, z, t)}{\partial y}\right|_{x=L_{y}}=0, \\
\left.\frac{\partial C(x, y, z, t)}{\partial z}\right|_{z=0}=0,\left.\frac{\partial C(x, y, z, t)}{\partial z}\right|_{x=L_{z}}=0, C(x, y, z, 0)=f(x, y, z) .
\end{gathered}
$$

Boundary conditions correspond to absents of flow of dopant through external boundaries of the considered heterostructure. Initial distribution depends on type of doping: diffusion doping or ion doping. In the Eqs.(1) and (2) function $C(x, y, z, t)$ describes the spatio-temporal distribution of concentration of dopant; $T$ is the temperature of annealing; $D_{C}$ is the dopant diffusion coefficient. Value of dopant diffusion coefficient could be changed by heating and cooling of heterostructure (with account Arrhenius law), radiation processing of materials of heterostructure and high doping of materials. Approximation of dopant diffusion coefficient on parameters could be written as [19-21]

$$
D_{C}=D_{L}(x, y, z, T)\left[1+\xi \frac{C^{\gamma}(x, y, z, t)}{P^{\gamma}(x, y, z, T)}\right]\left[1+\varsigma_{1} \frac{V(x, y, z, t)}{V^{*}}+\varsigma_{2} \frac{V^{2}(x, y, z, t)}{\left(V^{*}\right)^{2}}\right] .
$$

Here function $D_{L}(x, y, z, T)$ takes into account the spatial (due to presents several layers in heterostructure) and temperature (due to Arrhenius law) dependences of dopant diffusion coefficient. Function $P(x, y, z, T)$ describes approximation of the limit of solubility of dopant on coordinates in heterostructure and temperature. The parameter $\gamma$ shows averaged quantity of charged defects, which interacting which interacting with atoms of dopant [19]. The function $V(x, y, z, t)$ describes the spatio-temporal distribution of concentration of radiation vacancies; $V^{*}$ is the equilibrium distribution of concentration of vacancies. The second term of the last part of Eq.(3) taking into account generation of radiation vacancies. The third term of the last part of Eq.(3) taking into account generation of radiation divacancies. Dependence of dopant diffusion coefficient on concentration of dopant has been described in details in [19]. It should be noted, that using diffusion type of doping did not generation radiation defects. In this situation $\zeta_{1}=\zeta_{2}=0$. We determine spatiotemporal distributions of concentrations of radiation defects by solving the following system of equations [20,21]

$$
\begin{aligned}
& \frac{\partial I(x, y, z, t)}{\partial t}=\frac{\partial}{\partial x}\left[D_{I}(x, y, z, T) \frac{\partial I(x, y, z, t)}{\partial x}\right]+\frac{\partial}{\partial y}\left[D_{I}(x, y, z, T) \frac{\partial I(x, y, z, t)}{\partial y}\right]-k_{I, I}(x, y, z, T) \times \\
& \quad \times I^{2}(x, y, z, t)+\frac{\partial}{\partial z}\left[D_{I}(x, y, z, T) \frac{\partial I(x, y, z, t)}{\partial z}\right]-k_{I, V}(x, y, z, T) I(x, y, z, t) V(x, y, z, t) \quad(4) \\
& \frac{\partial V(x, y, z, t)}{\partial t}=\frac{\partial}{\partial x}\left[D_{V}(x, y, z, T) \frac{\partial V(x, y, z, t)}{\partial x}\right]+\frac{\partial}{\partial y}\left[D_{V}(x, y, z, T) \frac{\partial V(x, y, z, t)}{\partial y}\right]-k_{V, V}(x, y, z, T) \times \\
& \quad \times V^{2}(x, y, z, t)+\frac{\partial}{\partial z}\left[D_{V}(x, y, z, T) \frac{\partial V(x, y, z, t)}{\partial z}\right]-k_{I, V}(x, y, z, T) I(x, y, z, t) V(x, y, z, t) .
\end{aligned}
$$


Terms in the right sides of Eqs.(4) with derivatives on coordinate gives a possibility to take into account diffusion of point radiation defects. Terms with $V^{2}(x, y, z, t)$ and $I^{2}(x, y, z, t)$ gives a possibility to take into account divacancies and diinterstitials. Term with $I(x, y, z, t) V(x, y, z, t)$ gives a possibility to take into account recombination of point radiation defects. Boundary and initial conditions for Eqs.(4) are

$$
\begin{gathered}
\left.\frac{\partial \rho(x, y, z, t)}{\partial x}\right|_{x=0}=0,\left.\frac{\partial \rho(x, y, z, t)}{\partial x}\right|_{x=L_{x}}=0,\left.\frac{\partial \rho(x, y, z, t)}{\partial y}\right|_{y=0}=0,\left.\frac{\partial \rho(x, y, z, t)}{\partial y}\right|_{y=L_{y}}=0, \\
\left.\frac{\partial \rho(x, y, z, t)}{\partial z}\right|_{z=0}=0,\left.\frac{\partial \rho(x, y, z, t)}{\partial z}\right|_{z=L_{z}}=0, \rho(x, y, z, 0)=f_{\rho}(x, y, z) .
\end{gathered}
$$

Here $\rho=I, V$. The function $I(x, y, z, t)$ describes the spatio-temporal distribution of concentration of radiation interstitials. The functions $D_{\rho}(x, y, z, T)$ describe the diffusion coefficients of point radiation defects. The function $k_{I, V}(x, y, z, T)$ describes the parameter of recombination of point radiation defects. The functions $k_{\rho, \rho}(x, y, z, T)$ describe the parameters of generation of simplest complexes of point radiation defects.

Spatio-temporal distributions of concentrations of divacancies $\Phi_{\rho}(x, y, z, t)$ have been calculated by solving the following system of equations $[20,21]$

$$
\begin{aligned}
& \frac{\partial \Phi_{I}(x, y, z, t)}{\partial t}=\frac{\partial}{\partial x}\left[D_{\Phi I}(x, y, z, T) \frac{\partial \Phi_{I}(x, y, z, t)}{\partial x}\right]+\frac{\partial}{\partial y}\left[D_{\Phi I}(x, y, z, T) \frac{\partial \Phi_{I}(x, y, z, t)}{\partial y}\right]+ \\
& +\frac{\partial}{\partial z}\left[D_{\Phi I}(x, y, z, T) \frac{\partial \Phi_{I}(x, y, z, t)}{\partial z}\right]+k_{I, I}(x, y, z, T) I^{2}(x, y, z, t)-k_{I}(x, y, z, T) I(x, y, z, t) \\
& \frac{\partial \Phi_{V}(x, y, z, t)}{\partial t}=\frac{\partial}{\partial x}\left[D_{\Phi V}(x, y, z, T) \frac{\partial \Phi_{V}(x, y, z, t)}{\partial x}\right]+\frac{\partial}{\partial y}\left[D_{\Phi V}(x, y, z, T) \frac{\partial \Phi_{V}(x, y, z, t)}{\partial y}\right]+ \\
& +\frac{\partial}{\partial z}\left[D_{\Phi V}(x, y, z, T) \frac{\partial \Phi_{V}(x, y, z, t)}{\partial z}\right]+k_{V, V}(x, y, z, T) V^{2}(x, y, z, t)-k_{V}(x, y, z, T) V(x, y, z, t) .
\end{aligned}
$$

Terms in the right sides of Eqs.(6) with derivatives on coordinate gives a possibility to take into account diffusion of complexes of point radiation defects. Terms with $V^{2}(x, y, z, t)$ and $I^{2}(x, y, z, t)$ gives a possibility to take into account divacancies and diinterstitials. Term with $I(x, y, z, t)$ and $V$ $(x, y, z, t)$ gives a possibility to take into account decay of complexes of point radiation defects. Boundary and initial conditions for Eqs.(4) are

$$
\begin{gathered}
\left.\frac{\partial \Phi_{\rho}(x, y, z, t)}{\partial x}\right|_{x=0}=0,\left.\frac{\partial \Phi_{\rho}(x, y, z, t)}{\partial x}\right|_{x=L_{x}}=0,\left.\frac{\partial \Phi_{\rho}(x, y, z, t)}{\partial y}\right|_{y=0}=0,\left.\frac{\partial \Phi_{\rho}(x, y, z, t)}{\partial y}\right|_{y=L_{y}}=0, \\
\left.\frac{\partial \Phi_{\rho}(x, y, z, t)}{\partial z}\right|_{z=0}=0,\left.\frac{\partial \Phi_{\rho}(x, y, z, t)}{\partial z}\right|_{z=L_{z}}=0, \Phi_{I}(x, y, z, 0)=f_{\Phi I}(x, y, z), \Phi_{V}(x, y, z, 0)=f_{\Phi V}(x, y, z) .(7)
\end{gathered}
$$


Here $D_{\Phi \rho}(x, y, z, T)$ are the diffusion coefficients of the above complexes of radiation defects; $k_{I}(x, y, z, T)$ and $k_{V}(x, y, z, T)$ are the parameters of decay of these complexes. To determine spatiotemporal distribution of concentration of dopant we transform the Eq.(1) to the following integrodifferential form

$$
\begin{gathered}
\frac{x y z}{L_{x} L_{y} L_{z}} \int_{L_{x} L_{y} L_{z}}^{x} \int_{z}^{z} C(u, v, w, t) d w d v d u=\frac{y z}{L_{y} L_{z}} \int_{0}^{t} \int_{L_{y} L_{z}}^{y} \int^{z}\left[1+\varsigma_{1} \frac{V(x, v, w, \tau)}{V^{*}}+\varsigma_{2} \frac{V^{2}(x, v, w, \tau)}{\left(V^{*}\right)^{2}}\right] \times \\
\times D_{L}(x, v, w, T)\left[1+\xi \frac{C^{\gamma}(x, v, w, \tau)}{P^{\gamma}(x, v, w, T)}\right] \frac{\partial C(x, v, w, \tau)}{\partial x} d \tau+\frac{x z}{L_{x} L_{z}} \int_{0}^{t} \int_{L_{x} L_{z}}^{x} \int^{z} D_{L}(u, y, w, T) \times \\
\times\left[1+\varsigma_{1} \frac{V(u, y, w, \tau)}{V^{*}}+\varsigma_{2} \frac{V^{2}(u, y, w, \tau)}{\left(V^{*}\right)^{2}}\right]\left[1+\xi \frac{C^{\gamma}(u, y, w, \tau)}{P^{\gamma}(x, y, z, T)}\right] \frac{\partial C(u, y, w, \tau)}{\partial y} d \tau+ \\
+\frac{x y}{L_{x} L_{y}} \int_{0}^{t} \int_{L_{x} L_{y}}^{x} \int_{L}^{y} D_{L}(u, v, z, T)\left[1+\varsigma_{1} \frac{V(u, v, z, \tau)}{V^{*}}+\varsigma_{2} \frac{V^{2}(u, v, z, \tau)}{\left(V^{*}\right)^{2}}\right]\left[1+\xi \frac{C^{\gamma}(u, v, z, \tau)}{P^{\gamma}(x, y, z, T)}\right] \times \\
\times \frac{\partial C(u, v, z, \tau)}{\partial z} d \tau+\frac{x y z}{L_{x} L_{y} L_{z}} \int_{L_{x} L_{y} L_{z}}^{x} \int^{y} f(u, v, w) d w d v d u .
\end{gathered}
$$

We determine solution of the above equation by using Bubnov-Galerkin approach [22]. Framework the approach we determine solution of the Eq.(1a) as the following series

$$
C_{0}(x, y, z, t)=\sum_{n=0}^{N} a_{n C} c_{n}(x) c_{n}(y) c_{n}(z) e_{n C}(t)
$$

where $e_{n C}(t)=\exp \left[-\pi^{2} n^{2} D_{0 c} t\left(L_{x}^{-2}+L_{y}^{-2}+L_{z}^{-2}\right)\right], c_{n}(\chi)=\cos \left(\pi n \chi / L_{\chi}\right)$. The above series includes into itself finite number of terms $N$. The considered series is similar with solution of linear Eq.(1) (i.e. with $\xi=0$ ) and averaged dopant diffusion coefficient $D_{0}$. Substitution of the series into Eq.(1a) leads to the following result

$$
\begin{aligned}
& \frac{x y z}{\pi^{2}} \sum_{n=1}^{N} \frac{a_{C}}{n^{3}} s_{n}(x) s_{n}(y) s_{n}(z) e_{n C}(t)=-\frac{y z}{L_{y} L_{z}} \int_{0}^{t} \int_{L_{y}}^{y} \int_{L_{z}}^{z}\left\{1+\left[\sum_{n=1}^{N} a_{n C} c_{n}(x) c_{n}(v) c_{n}(w) e_{n C}(\tau)\right]^{\gamma} \times\right. \\
& \left.\times \frac{\xi}{P^{\gamma}(x, v, w, T)}\right\}\left[1+\varsigma_{1} \frac{V(x, v, w, \tau)}{V^{*}}+\varsigma_{2} \frac{V^{2}(x, v, w, \tau)}{\left(V^{*}\right)^{2}}\right] D_{L}(x, v, w, T) \sum_{n=1}^{N} a_{n C} s_{n}(x) c_{n}(v) \times \\
& \times n c_{n}(w) e_{n C}(\tau) d \tau-\frac{x z}{L_{x} L_{z}} \int_{0}^{t} \int_{L_{x} L_{z}}^{x}\left\{\left\{1+\left[\sum_{m=1}^{N} a_{m C} c_{m}(u) c_{m}(y) c_{m}(w) e_{m C}(\tau)\right]^{\gamma} \frac{\xi}{P^{\gamma}(u, y, w, T)}\right\} \times\right. \\
& \times D_{L}(u, y, w, T)\left[1+\varsigma_{1} \frac{V(u, y, w, \tau)}{V^{*}}+\varsigma_{2} \frac{V^{2}(u, y, w, \tau)}{\left(V^{*}\right)^{2}}\right] \sum_{n=1}^{N} n c_{n}(u) s_{n}(y) c_{n}(w) e_{n C}(\tau) d \tau \times \\
& \times a_{n C}-\frac{x y}{L_{x} L_{y}} \int_{0}^{t} \int_{L_{x} L_{y}}^{x} \int_{L}^{y} D_{L}(u, v, z, T)\left\{1+\frac{\xi}{P^{\gamma}(u, v, z, T)}\left[\sum_{n=1}^{N} a_{n C} c_{n}(u) c_{n}(v) c_{n}(z) e_{n C}(\tau)\right]^{\gamma}\right\} \times \\
& \times\left[1+\varsigma_{1} \frac{V(u, v, z, \tau)}{V^{*}}+\varsigma_{2} \frac{V^{2}(u, v, z, \tau)}{\left(V^{*}\right)^{2}}\right]_{n=1}^{N} n a_{n C} c_{n}(u) c_{n}(v) s_{n}(z) e_{n C}(\tau) d \tau+\frac{x y z}{L_{x} L_{y} L_{z}} \times
\end{aligned}
$$




$$
\times \int_{L_{x} L_{y} L_{z}}^{x} \int_{z}^{z} f(u, v, w) d w d v d u
$$

where $s_{n}(\chi)=\sin \left(\pi n \chi / L_{\chi}\right)$. We determine coefficients $a_{n}$ by using orthogonality condition of terms of the considered series framework scale of heterostructure. The condition gives us possibility to obtain relations for calculation of parameters $a_{n}$ for any quantity of terms $N$. In the common case the relations could be written as

$$
\begin{aligned}
& -\frac{L_{x}^{2} L_{y}^{2} L_{z}^{2}}{\pi^{5}} \sum_{n=1}^{N} \frac{a_{n C}}{n^{6}} e_{n C}(t)=-\frac{L_{y} L_{z}}{2 \pi^{2}} \int_{0}^{t} \int_{0}^{L_{x} L_{y} L_{2}} \int_{0} \int_{0} D_{L}(x, y, z, T)\left\{1+\left[\sum_{n=1}^{N} a_{n C} c_{n}(x) c_{n}(y) c_{n}(z) e_{n C}(\tau)\right]^{\gamma} \times\right. \\
& \left.\times \frac{\xi}{P^{\gamma}(x, y, z, T)}\right\}\left[1+\varsigma_{1} \frac{V(x, y, z, \tau)}{V^{*}}+\varsigma_{2} \frac{V^{2}(x, y, z, \tau)}{\left(V^{*}\right)^{2}} \sum_{n=1}^{N} \frac{a_{n C}}{n} s_{n}(2 x) c_{n}(y) c_{n}(z) e_{n C}(\tau) \times\right. \\
& \times\left\{y s_{n}(y)+\frac{L_{y}}{\pi n}\left[c_{n}(y)-1\right]\right\}\left\{z s_{n}(z)+\frac{L_{z}}{\pi n}\left[c_{n}(z)-1\right]\right\} d z d y d x d \tau-\int_{0}^{t} \int_{0}^{L} \int_{0}^{L_{y} L_{z}} \int_{0} \int_{0} D_{L}(x, y, z, T) \times \\
& \times D_{L}(x, y, z, T)\left\{1+\left[\sum_{n=1}^{N} a_{n C} c_{n}(x) c_{n}(y) c_{n}(z) e_{n C}(\tau)\right]^{\gamma} \frac{\xi}{P^{\gamma}(x, y, z, T)}\right\}\left[1+\varsigma_{1} \frac{V(x, y, z, \tau)}{V^{*}}+\right. \\
& \left.+\varsigma_{2} \frac{V^{2}(x, y, z, \tau)}{\left(V^{*}\right)^{2}}\right]\left[1+\varsigma_{1} \frac{V(x, y, z, \tau)}{V^{*}}+\varsigma_{2} \frac{V^{2}(x, y, z, \tau)}{\left(V^{*}\right)^{2}}\right]_{n=1}^{N}\left\{x s_{n}(x)+\frac{L_{x}}{\pi n}\left[c_{n}(x)-1\right]\right\} \frac{a_{n C}}{n} \times \\
& \times \frac{L_{x} L_{z}}{2 \pi^{2}} c_{n}(x) s_{n}(2 y) c_{n}(z) e_{n C}(\tau)\left\{z s_{n}(z)+\frac{L_{z}}{\pi n}\left[c_{n}(z)-1\right]\right\} d z d y d x d \tau-\frac{L_{x} L_{y}}{2 \pi^{2}} \times \\
& \times \int_{0}^{t} \int_{0}^{L_{x} L_{y} L_{z}} \int_{0} \int_{0}\left\{1+\left[\sum_{n=1}^{N} a_{n C} c_{n}(x) c_{n}(y) c_{n}(z) e_{n C}(\tau)\right]^{\gamma} \frac{\xi}{P^{\gamma}(x, y, z, T)}\right\}\left[1+\varsigma_{2} \frac{V^{2}(x, y, z, \tau)}{\left(V^{*}\right)^{2}}+\right. \\
& \left.+\varsigma_{1} \frac{V(x, y, z, \tau)}{V^{*}}\right] D_{L}(x, y, z, T) \sum_{n=1}^{N} \frac{a_{n C}}{n} c_{n}(x) c_{n}(y) s_{n}(z)\left\{x s_{n}(x)+\frac{L_{x}}{\pi n}\left[c_{n}(x)-1\right]\right\} \times \\
& \times\left\{y s_{n}(y)+\frac{L_{y}}{\pi n}\left[c_{n}(y)-1\right]\right\} e_{n C}(\tau) d z d y d x d \tau+\sum_{n=1}^{N} \int_{0}^{L_{x}}\left\{x s_{n}(x)+\frac{L_{x}}{\pi n}\left[c_{n}(x)-1\right]\right\} \times \\
& \times \int_{0}^{L_{y}}\left\{y s_{n}(y)+\frac{L_{y}}{\pi n}\left[c_{n}(y)-1\right]\right\} \int_{0}^{L_{z}}\left\{z s_{n}(z)+\frac{L_{z}}{\pi n}\left[c_{n}(z)-1\right]\right\} f(x, y, z) d z d y d x .
\end{aligned}
$$

As an example for $\gamma=0$ we obtain

$$
\begin{aligned}
& a_{n C}=\int_{0}^{L_{x} L_{y}}\left\{\{ y s _ { n } ( y ) + \frac { L _ { y } } { \pi n } [ c _ { n } ( y ) - 1 ] \} \int _ { 0 } ^ { L _ { z } } \{ z s _ { n } ( z ) + \frac { L _ { y } } { \pi n } [ c _ { n } ( z ) - 1 ] \} f ( x , y , z ) d z d y \left\{x s_{n}(x)+\right.\right. \\
& \left.\times\left[c_{n}(x)-1\right] \frac{L_{x}}{\pi n}\right\} d x\left(\frac { n } { 2 } \left\{\int_{0}^{t} \int_{0}^{L_{x}} s_{n}(2 x) \int_{0}^{L_{y}} c_{n}(y)\left\{y s_{n}(y)+\frac{L_{y}}{\pi n}\left[c_{n}(y)-1\right]\right\} \int_{0}^{L_{z}} D_{L}(x, y, z, T) \times\right.\right. \\
& \times\left\{z s_{n}(z)+\frac{L_{y}}{\pi n}\left[c_{n}(z)-1\right]\right\}\left[1+\varsigma_{1} \frac{V(x, y, z, \tau)}{V^{*}}+\varsigma_{2} \frac{V^{2}(x, y, z, \tau)}{\left(V^{*}\right)^{2}}\right]\left[1+\frac{\xi}{P^{\gamma}(x, y, z, T)}\right] \times
\end{aligned}
$$




$$
\begin{aligned}
& \times c_{n}(z) d z d y d x e_{n C}(\tau) d \tau+\int_{0}^{t} e_{n C}(\tau) \int_{0}^{L_{x}} c_{n}(x)\left\{x s_{n}(x)+\frac{L_{y}}{\pi n}\left[c_{n}(x)-1\right]\right\} \int_{0}^{L_{y}} s_{n}(2 y) \int_{0}^{L_{z}} c_{n}(z) \times \\
& \times\left\{z s_{n}(z)+\frac{L_{y}}{\pi n}\left[c_{n}(z)-1\right]\right\}\left[1+\frac{\xi}{P^{\gamma}(x, y, z, T)}\right]\left[1+\varsigma_{1} \frac{V(x, y, z, \tau)}{V^{*}}+\varsigma_{2} \frac{V^{2}(x, y, z, \tau)}{\left(V^{*}\right)^{2}}\right] \times \\
& \times D_{L}(x, y, z, T) d z d y d x d \tau+\int_{0}^{t} e_{n C}(\tau) \int_{0}^{L_{x}} c_{n}(x)\left\{x s_{n}(x)+\frac{L_{x}}{\pi n}\left[c_{n}(x)-1\right]\right\} \int_{0}^{L_{y}} c_{n}(y)\left\{s_{n}(y) \times\right. \\
& \times y+\frac{L_{y}}{\pi n}\left[c_{n}(y)-1\right] \int_{0}^{L_{z}} s_{n}(2 z) D_{L}(x, y, z, T)\left[1+\frac{\xi}{P^{\gamma}(x, y, z, T)}\right]\left[1+\varsigma_{2} \frac{V^{2}(x, y, z, \tau)}{\left(V^{*}\right)^{2}}+\right. \\
& \left.\left.\left.+\varsigma_{1} \frac{V(x, y, z, \tau)}{V^{*}}\right] d z d y d x d \tau\right\}-\frac{L_{z}^{2} L_{z}^{2} L_{z}^{2}}{\pi^{5} n^{6}} e_{n C}(t)\right)^{-1} \cdot
\end{aligned}
$$

For $\gamma=1$ one can obtain the following relation to determine required parameters

$$
a_{n C}=-\frac{\beta_{n}}{2 \alpha_{n}} \pm \sqrt{\beta_{n}^{2}+4 \alpha_{n} \int_{0}^{L_{x}} c_{n}(x) \int_{0}^{L_{y}} c_{n}(y) \int_{0}^{L_{z}} c_{n}(z) f(x, y, z) d z d y d x}
$$

where $\alpha_{n}=\frac{\xi L_{y} L_{z}}{2 \pi^{2} n} \int_{0}^{t} e_{n C}(\tau) \int_{0}^{L_{x}} s_{n}(2 x) \int_{0}^{L_{y}} \int_{0}^{L_{z}}\left[1+\varsigma_{1} \frac{V(x, y, z, \tau)}{V^{*}}+\varsigma_{2} \frac{V^{2}(x, y, z, \tau)}{\left(V^{*}\right)^{2}}\right] \frac{D_{L}(x, y, z, T)}{P(x, y, z, T)} \times$ $\times c_{n}(y) c_{n}(z)\left\{y s_{n}(y)+\frac{L_{y}}{\pi n}\left[c_{n}(y)-1\right]\right\}\left\{z s_{n}(z)+\frac{L_{z}}{\pi n}\left[c_{n}(z)-1\right]\right\} d z d y d x d \tau+\frac{\xi L_{x} L_{z}}{2 \pi^{2} n} \int_{0}^{t} e_{n C}(\tau) \times$ $\times \int_{0}^{L_{x}}\left\{x s_{n}(x)+\frac{L_{x}}{\pi n}\left[c_{n}(x)-1\right]\right\} \int_{0}^{L_{y} L_{z}}\left\{z s_{0}(z)-\frac{L_{z}}{\pi n}\left[c_{n}(z)-1\right]\right\}\left[1+\varsigma_{1} \frac{V(x, y, z, \tau)}{V^{*}}+\varsigma_{2} \frac{V^{2}(x, y, z, \tau)}{\left(V^{*}\right)^{2}}\right] \times$ $\times c_{n}(x) s_{n}(2 y) c_{n}(z) \frac{D_{L}(x, y, z, T)}{P(x, y, z, T)} d z d y d x d \tau+\frac{\xi L_{x} L_{y}}{2 \pi^{2} n} \int_{0}^{t} e_{n C}(\tau) \int_{0}^{L_{x}} c_{n}(x) \int_{0}^{L_{y}} c_{n}(y) \int_{0}^{L_{z}} \frac{D_{L}(x, y, z, T)}{P(x, y, z, T)} \times$ $\times\left[1+\varsigma_{1} \frac{V(x, y, z, \tau)}{V^{*}}+\varsigma_{2} \frac{V^{2}(x, y, z, \tau)}{\left(V^{*}\right)^{2}}\right]\left\{x s_{n}(x)+\frac{L_{x}}{\pi n}\left[c_{n}(x)-1\right]\right\}\left\{y s_{n}(y)+\frac{L_{y}}{\pi n}\left[c_{n}(y)-1\right]\right\} d y \times$ $\times s_{n}(2 z) d z d x d \tau, \beta_{n}=\frac{L_{y} L_{z}}{2 n \pi^{2}} \int_{0}^{t} e_{n C}(\tau) \int_{0}^{L_{x}} s_{n}(2 x) \int_{0}^{L_{y} L_{z}} \int_{0} c_{n}(z)\left[1+\varsigma_{1} \frac{V(x, y, z, \tau)}{V^{*}}+\varsigma_{2} \frac{V^{2}(x, y, z, \tau)}{\left(V^{*}\right)^{2}}\right] \times$ $\times D_{L}(x, y, z, T)\left\{z s_{n}(z)+\frac{L_{z}}{\pi n}\left[c_{n}(z)-1\right]\right\} d z c_{n}(y)\left\{y s_{n}(y)+\frac{L_{y}}{\pi n}\left[c_{n}(y)-1\right]\right\} d y d x d \tau+\frac{L_{x} L_{z}}{2 n \pi^{2}} \times$ $\times \int_{0}^{t} e_{n C}(\tau) \int_{0}^{L_{x}} c_{n}(x)\left\{x s_{n}(x)+\frac{L_{x}}{\pi n}\left[c_{n}(x)-1\right]\right\} \int_{0}^{L_{y}} s_{n}(2 y) \int_{0}^{L_{z}} c_{n}(z)\left[1+\varsigma_{1} \frac{V(x, y, z, \tau)}{V^{*}}+\varsigma_{2} \frac{V^{2}(x, y, z, \tau)}{\left(V^{*}\right)^{2}}\right] \times$ $\times D_{L}(x, y, z, T)\left\{z s_{n}(z)+\frac{L_{z}}{\pi n}\left[c_{n}(z)-1\right]\right\} d z d y d x d \tau+\frac{L_{x} L_{y}}{2 n \pi^{2}} \int_{0}^{t} e_{n C}(\tau) \int_{0}^{L_{x}}\left\{x s_{n}(x)+\frac{L_{x}}{\pi n}\left[c_{n}(x)-1\right]\right\} \times$ $\times \int_{0}^{L_{y}}\left\{y s_{n}(y)+\frac{L_{y}}{\pi n}\left[c_{n}(y)-1\right]\right\} \int_{0}^{L_{z}} s_{n}(2 z) D_{L}(x, y, z, T)\left[1+\varsigma_{1} \frac{V(x, y, z, \tau)}{V^{*}}+\varsigma_{2} \frac{V^{2}(x, y, z, \tau)}{\left(V^{*}\right)^{2}}\right] d z \times$ 
$\times c_{n}(y) d y c_{n}(x) d x d \tau-L_{x}^{2} L_{y}^{2} L_{z}^{2} e_{n C}(t) / \pi^{5} n^{6}$.

Analogous way could be used to calculate values of parameters $a_{n}$ for larger values of parameter $\gamma$. However the relations will not be present in the paper because the relations are bulky.Equations of the system (4) have been also solved by using Bubnov-Galerkin approach. Previously we transform the differential equations to the following integro- differential form

$$
\begin{aligned}
& \frac{x y z}{L_{x} L_{y} L_{z}} \int_{L_{x} L_{y}}^{x} \int_{L_{z}}^{y} \int_{z}^{z} I(u, v, w, t) d w d v d u=\int_{0}^{t} \int_{L_{y}}^{y} \int_{L_{z}}^{z} D_{I}(x, v, w, T) \frac{\partial I(x, v, w, \tau)}{\partial x} d w d v d \tau \times \\
& \times \frac{y z}{L_{y} L_{z}}+\frac{x z}{L_{x} L_{z}} \int_{0}^{t} \int_{L_{x} L_{z}}^{x} \int_{I}^{z} D_{I}(u, y, w, T) \frac{\partial I(u, y, w, \tau)}{\partial x} d w d u d \tau-\int_{L_{x} L_{y}}^{x} \int_{L_{z}}^{z} k_{I, V}^{z}(u, v, w, T) \times \\
& \times I(u, v, w, t) V(u, v, w, t) d w d v d u \frac{x y z}{L_{x} L_{y} L_{z}}+\frac{x y}{L_{x} L_{y}} \int_{0}^{t} \int_{L_{x} L_{y}}^{x} \int_{y} \frac{\partial I(u, v, z, \tau)}{\partial z} \times \\
& \times D_{I}(u, v, z, T) d v d u d \tau-\frac{x y z}{L_{x} L_{y} L_{z}} \int_{L_{x} L_{y}}^{x} \int_{L_{z}}^{z} k_{I, I}(u, v, w, T) I^{2}(u, v, w, t) d w d v d u+ \\
& +\frac{x y z}{L_{x} L_{y} L_{z}} \int_{L_{x}}^{x} \int_{L_{y}}^{y} \int_{L_{z}}^{z} f_{I}(u, v, w) d w d v d u \\
& \frac{x y z}{L_{x} L_{y} L_{z}} \int_{L_{x} L_{y}}^{x} \int_{L_{z}}^{z} \int_{z} V(u, v, w, t) d w d v d u=\int_{0}^{t} \int_{L_{y}}^{y} \int_{L_{z}}^{z} D_{V}(x, v, w, T) \frac{\partial V(x, v, w, \tau)}{\partial x} d w d v d \tau \times \\
& \times \frac{y z}{L_{y} L_{z}}+\frac{x z}{L_{x} L_{z}} \int_{0}^{t} \int_{L_{x} L_{z}}^{x} \int_{V}^{z} D_{V}(u, y, w, T) \frac{\partial V(u, y, w, \tau)}{\partial x} d w d u d \tau+\int_{0}^{t} \int_{L_{x} L_{y}}^{y} D_{V}(u, v, z, T) \times \\
& \times \frac{\partial V(u, v, z, \tau)}{\partial z} d v d u d \tau \frac{x y}{L_{x} L_{y}}-\int_{L_{x} L_{y} L_{z}}^{x} \int_{I, V}^{z} k_{I, v}(u, v, w, T) I(u, v, w, t) V(u, v, w, t) d w d v d u \times \\
& \times \frac{x y z}{L_{x} L_{y} L_{z}}+\frac{x y z}{L_{x} L_{y} L_{z}}\left[\int_{L_{x} L_{y} L_{z}}^{x} \int_{V}^{z} f_{V}(u, v, w) d w d v d u-\int_{L_{x} L_{y} L_{z}}^{x} \int_{V, V}^{z} k_{V}(u, v, w, T) V^{2}(u, v, w, t) d w d v d u\right] .
\end{aligned}
$$

We determine solutions of the Eqs. $(4 a)$ as the following series

$$
\rho_{0}(x, y, z, t)=\sum_{n=1}^{N} a_{n \rho} c_{n}(x) c_{n}(y) c_{n}(z) e_{n \rho}(t) .
$$

Coefficients $a_{n \rho}$ are not yet known. Substitution of the series into Eqs.(4a) leads to the following results

$$
\begin{aligned}
& \frac{x y z}{\pi^{3}} \sum_{n=1}^{N} \frac{a_{n I}}{n^{3}} s_{n}(x) s_{n}(y) s_{n}(z) e_{n I}(t)=-\frac{y z \pi}{L_{x} L_{y} L_{z}} \sum_{n=1}^{N} a_{n I} \int_{0}^{t} \int_{L_{y}}^{y} c_{n}(y) \int_{L_{z}}^{z} c_{n}(z) D_{I}(x, v, w, T) d w d v \times \\
& \times e_{n I}(\tau) d \tau s_{n}(x)-\frac{x z \pi}{L_{x} L_{y} L_{z}} \sum_{n=1}^{N} a_{n I} s_{n}(y) \int_{0}^{t} e_{n I}(\tau) \int_{L_{x}}^{x} c_{n}(x) \int_{L_{z}}^{z} c_{n}(z) D_{I}(u, y, w, T) d w d u d \tau- \\
& -\frac{x y \pi}{L_{x} L_{y} L_{z}} \sum_{n=1}^{N} a_{n I} s_{n}(z) \int_{0}^{t} e_{n I}(\tau) \int_{L_{x}}^{x} c_{n}(x) \int_{L_{y}}^{y} c_{n}(y) D_{I}(u, v, z, T) d v d u d \tau-\int_{L_{x} L_{y} L_{z}}^{x} \int_{I, I}^{z} k_{I}(u, v, v, T) \times
\end{aligned}
$$




$$
\begin{aligned}
& \times\left[\sum_{n=1}^{N} a_{n I} c_{n}(u) c_{n}(v) c_{n}(w) e_{n I}(t)\right]^{2} d w d v d u \frac{x y z}{L_{x} L_{y} L_{z}}-\frac{x y z}{L_{x} L_{y} L_{z}} \int_{L_{x}}^{x} \int_{y} \int_{L_{z}}^{y} \sum_{n=1}^{N} a_{n I} c_{n}(u) c_{n}(v) c_{n}(w) \times \\
& \times e_{n I}(t) \sum_{n=1}^{N} a_{n V} c_{n}(u) c_{n}(v) c_{n}(w) e_{n V}(t) k_{I, V}(u, v, v, T) d w d v d u+\frac{x y z}{L_{x} L_{y} L_{z}} \int_{L_{x} L_{y} L_{z}}^{x} \int_{z}^{z} f_{I}(u, v, w) d w d v d u \\
& \frac{x y z}{\pi^{3}} \sum_{n=1}^{N} \frac{a_{n V}}{n^{3}} s_{n}(x) s_{n}(y) s_{n}(z) e_{n V}(t)=-\frac{y z \pi}{L_{x} L_{y} L_{z}} \sum_{n=1}^{N} a_{n V} \int_{0}^{t} \int_{L_{y}}^{y} c_{n}(y) \int_{L_{z}}^{z} c_{n}(z) D_{V}(x, v, w, T) d w d v \times \\
& \times e_{n V}(\tau) d \tau s_{n}(x)-\frac{x z \pi}{L_{x} L_{y} L_{z}} \sum_{n=1}^{N} a_{n V} s_{n}(y) \int_{0}^{t} e_{n V}(\tau) \int_{L_{x}}^{x} c_{n}(x) \int_{L_{z}}^{z} c_{n}(z) D_{V}(u, y, w, T) d w d u d \tau- \\
& -\frac{x y \pi}{L_{x} L_{y} L_{z}} \sum_{n=1}^{N} a_{n V} S_{n}(z) \int_{0}^{t} e_{n V}(\tau) \int_{L_{x}}^{x} c_{n}(x) \int_{L_{y}}^{y} c_{n}(y) D_{V}(u, v, z, T) d v d u d \tau-\int_{L_{x} L_{y} L_{z}}^{x} \int_{V, V}^{z} k_{V}(u, v, v, T) \times \\
& \times\left[\sum_{n=1}^{N} a_{n V} c_{n}(u) c_{n}(v) c_{n}(w) e_{n I}(t)\right]^{2} d w d v d u \frac{x y z}{L_{x} L_{y} L_{z}}-\frac{x y z}{L_{x} L_{y} L_{z}} \int_{L_{x}}^{x} \int_{L_{y} L_{z} n=1}^{y} \int^{N} a_{n I} c_{n}(u) c_{n}(v) c_{n}(w) \times \\
& \times e_{n I}(t) \sum_{n=1}^{N} a_{n V} c_{n}(u) c_{n}(v) c_{n}(w) e_{n V}(t) k_{I, V}(u, v, v, T) d w d v d u+\frac{x y z}{L_{x} L_{y} L_{z}} \int_{L_{x} L_{y} L_{z}}^{x} \int_{V}^{z} f_{V}(u, v, w) d w d v d u .
\end{aligned}
$$

We determine coefficients $a_{n \rho}$ by using orthogonality condition on the scale of heterostructure. The condition gives us possibility to obtain relations to calculate $a_{n \rho}$ for any quantity $N$ of terms of considered series. In the common case equations for the required coefficients could be written as

$$
\begin{aligned}
& -\frac{L_{x}^{2} L_{y}^{2} L_{z}^{2}}{\pi^{5}} \sum_{n=1}^{N} \frac{a_{n I}}{n^{6}} e_{n I}(t)=-\frac{1}{2 \pi L_{x}} \sum_{n=1}^{N} \frac{a_{n I}}{n^{2}} \int_{0}^{t} \int_{0}^{L_{x}}\left[1-c_{n}(2 x)\right] \int_{0}^{L_{y}}\left\{L_{y}+y s_{n}(2 y)+\frac{L_{y}}{2 \pi n}\left[c_{n}(2 y)-1\right]\right\} \times \\
& \times \int_{0}^{L_{z}} D_{I}(x, y, z, T)\left\{z s_{n}(z)+\frac{L_{z}}{2 \pi n}\left[c_{n}(z)-1\right]\right\} d z d y d x e_{n I}(\tau) d \tau-\frac{1}{2 \pi L_{y}} \sum_{n=1}^{N} \frac{a_{n I}}{n^{2}} \int_{0}^{t} \int_{0}^{L_{x}}\left\{x s_{n}(2 x)+\right. \\
& \left.+L_{x}+\frac{L_{x}}{\pi n}\left[c_{n}(2 x)-1\right]\right\} \int_{0}^{L_{\nu} L_{z}} \int_{0} D_{I}(x, y, z, T)\left\{L_{z}+z s_{n}(2 z)+\frac{L_{z}}{2 \pi n}\left[c_{n}(2 z)-1\right]\right\} d z\left[1-c_{n}(2 y)\right] \times \\
& \times d y d x e_{n I}(\tau) d \tau \int_{0}^{L_{z}} D_{I}(x, y, z, T)\left\{L_{z}+z s_{n}(2 z)+\frac{L_{z}}{2 \pi n}\left[c_{n}(2 z)-1\right]\right\} d z d y d x e_{n I}(\tau) d \tau- \\
& -\frac{1}{2 \pi L_{z}} \sum_{n=1}^{N} \frac{a_{n I}}{n^{2}} \int_{0}^{t} \int_{0}^{L_{x}}\left\{L_{x}+x s_{n}(2 x)+\frac{L_{x}}{2 \pi n}\left[c_{n}(2 x)-1\right] \int_{0}^{L_{y}}\left\{L_{y}+y s_{n}(2 y)+\frac{L_{y}}{2 \pi n}\left[c_{n}(2 y)-1\right]\right\} \times\right. \\
& \times \int_{0}^{L_{2}}\left[1-c_{n}(2 z)\right] D_{I}(x, y, z, T) d z d y d x e_{n I}(\tau) d \tau-\sum_{n=1}^{N} a_{n I}^{2} e_{n I}(2 t) \int_{0}^{L_{x}}\left\{L_{x}+\frac{L_{x}}{2 \pi n}\left[c_{n}(2 x)-1\right]+\right. \\
& \left.+x s_{n}(2 x)\right\} \int_{0}^{L_{y}}\left\{L_{y}+y s_{n}(2 y)+\frac{L_{y}}{2 \pi n}\left[c_{n}(2 y)-1\right]\right\} \int_{0}^{L_{z}} k_{I, I}(x, y, z, T)\left\{L_{z}+\frac{L_{z}}{2 \pi n}\left[c_{n}(2 z)-1\right]+\right. \\
& \left.+z s_{n}(2 z)\right\} d z d y d x-\sum_{n=1}^{N} a_{n I} a_{n V} e_{n I}(t) e_{n V}(t) \int_{0}^{L_{x}}\left\{L_{x}+x s_{n}(2 x)+\frac{L_{x}}{2 \pi n}\left[c_{n}(2 x)-1\right]\right\} \int_{0}^{L_{y}}\left\{L_{y}+\right.
\end{aligned}
$$




$$
\begin{aligned}
& \left.+y s_{n}(2 y)+\frac{L_{y}}{2 \pi n}\left[c_{n}(2 y)-1\right]\right\} \int_{0}^{L_{z}} k_{I, V}(x, y, z, T)\left\{L_{z}+z s_{n}(2 z)+\frac{L_{z}}{2 \pi n}\left[c_{n}(2 z)-1\right]\right\} d z \times \\
& \times d y d x+\sum_{n=1}^{N} \int_{0}^{L_{x}}\left\{x s_{n}(x)+\frac{L_{x}}{\pi n}\left[c_{n}(x)-1\right]\right\} \int_{0}^{L_{y}}\left\{y s_{n}(y)+\frac{L_{y}}{\pi n}\left[c_{n}(y)-1\right]\right\} \int_{0}^{L_{z}} f_{I}(x, y, z, T) \times \\
& \times\left\{L_{z}+z s_{n}(2 z)+\frac{L_{z}}{2 \pi n}\left[c_{n}(2 z)-1\right]\right\} d z d y d x \\
& -\frac{L_{x}^{2} L_{y}^{2} L_{z}^{2}}{\pi^{5}} \sum_{n=1}^{N} \frac{a_{n V}}{n^{6}} e_{n V}(t)=-\frac{1}{2 \pi L_{x}} \sum_{n=1}^{N} \frac{a_{n V}}{n^{2}} \int_{0}^{t} \int_{0}^{L_{x}}\left[1-c_{n}(2 x)\right] \int_{0}^{L_{y}}\left\{L_{y}+y s_{n}(2 y)+\frac{L_{y}}{2 \pi n}\left[c_{n}(2 y)-1\right]\right\} \times \\
& \times \int_{0}^{L_{z}} D_{V}(x, y, z, T)\left\{z s_{n}(z)+\frac{L_{z}}{2 \pi n}\left[c_{n}(z)-1\right]\right\} d z d y d x e_{n V}(\tau) d \tau-\frac{1}{2 \pi L_{y}} \sum_{n=1}^{N} \frac{a_{n V}}{n^{2}} \int_{0}^{t} \int_{0}^{L_{x}}\left\{x s_{n}(2 x)+\right. \\
& \left.+L_{x}+\frac{L_{x}}{\pi n}\left[c_{n}(2 x)-1\right]\right\} \int_{0}^{L_{\nu} L_{z}} \int_{0} D_{V}(x, y, z, T)\left\{L_{z}+z s_{n}(2 z)+\frac{L_{z}}{2 \pi n}\left[c_{n}(2 z)-1\right]\right\} d z\left[1-c_{n}(2 y)\right] \times \\
& \times d y d x e_{n V}(\tau) d \tau \int_{0}^{L_{z}} D_{V}(x, y, z, T)\left\{L_{z}+z s_{n}(2 z)+\frac{L_{z}}{2 \pi n}\left[c_{n}(2 z)-1\right]\right\} d z d y d x e_{n V}(\tau) d \tau- \\
& -\frac{1}{2 \pi L_{z}} \sum_{n=1}^{N} \frac{a_{n V}}{n^{2}} \int_{0}^{t} \int_{0}^{L_{x}}\left\{L_{x}+x s_{n}(2 x)+\frac{L_{x}}{2 \pi n}\left[c_{n}(2 x)-1\right] \int_{0}^{L_{y}}\left\{L_{y}+y s_{n}(2 y)+\frac{L_{y}}{2 \pi n}\left[c_{n}(2 y)-1\right]\right\} \times\right. \\
& \times \int_{0}^{L_{z}}\left[1-c_{n}(2 z)\right] D_{V}(x, y, z, T) d z d y d x e_{n V}(\tau) d \tau-\sum_{n=1}^{N} a_{n V}^{2} e_{n V}(2 t) \int_{0}^{L_{x}}\left\{L_{x}+\frac{L_{x}}{2 \pi n}\left[c_{n}(2 x)-1\right]+\right. \\
& \left.+x s_{n}(2 x)\right\} \int_{0}^{L_{y}}\left\{L_{y}+y s_{n}(2 y)+\frac{L_{y}}{2 \pi n}\left[c_{n}(2 y)-1\right]\right\} \int_{0}^{L_{z}} k_{V, V}(x, y, z, T)\left\{L_{z}+\frac{L_{z}}{2 \pi n}\left[c_{n}(2 z)-1\right]+\right. \\
& \left.+z s_{n}(2 z)\right\} d z d y d x-\sum_{n=1}^{N} a_{n I} a_{n V} e_{n I}(t) e_{n V}(t) \int_{0}^{L_{x}}\left\{L_{x}+x s_{n}(2 x)+\frac{L_{x}}{2 \pi n}\left[c_{n}(2 x)-1\right]\right\} \int_{0}^{L_{y}}\left\{L_{y}+\right. \\
& +y s_{n}(2 y)+\frac{L_{y}}{2 \pi n}\left[c_{n}(2 y)-1\right] \int_{0}^{L_{z}} k_{I, V}(x, y, z, T)\left\{L_{z}+z s_{n}(2 z)+\frac{L_{z}}{2 \pi n}\left[c_{n}(2 z)-1\right]\right\} d z \times \\
& \times d y d x+\sum_{n=1}^{N} \int_{0}^{L_{x}}\left\{x s_{n}(x)+\frac{L_{x}}{\pi n}\left[c_{n}(x)-1\right]\right\} \int_{0}^{L_{y}}\left\{y s_{n}(y)+\frac{L_{y}}{\pi n}\left[c_{n}(y)-1\right]\right\} \int_{0}^{L_{z}} f_{V}(x, y, z, T) \times \\
& \times\left\{L_{z}+z s_{n}(2 z)+\frac{L_{z}}{2 \pi n}\left[c_{n}(2 z)-1\right]\right\} d z d y d x .
\end{aligned}
$$

In the final form relations for required parameters could be written as

$$
a_{n I}=-\frac{b_{3}+A}{4 b_{4}} \pm \sqrt{\frac{\left(b_{3}+A\right)^{2}}{4}-4 b_{4}\left(y+\frac{b_{3} y-\gamma_{n V} \lambda_{n I}^{2}}{A}\right)}, a_{n V}=-\frac{\gamma_{n I} a_{n I}^{2}+\delta_{n I} a_{n I}+\lambda_{n I}}{\chi_{n I} a_{n I}},
$$

where $\gamma_{n \rho}=e_{n \rho}(2 t) \int_{0}^{L_{x}} \int_{0}^{L_{y} L_{z}} \int_{0}^{k} k_{\rho, \rho}(x, y, z, T)\left\{L_{x}+x s_{n}(2 x)+\frac{L_{x}}{2 \pi n}\left[c_{n}(2 x)-1\right]\right\}\left\{y s_{n}(2 y)+L_{y}+\right.$ 


$$
\begin{aligned}
& \left.+\frac{L_{y}}{2 \pi n}\left[c_{n}(2 y)-1\right]\right\}\left\{L_{z}+z s_{n}(2 z)+\frac{L_{z}}{2 \pi n}\left[c_{n}(2 z)-1\right]\right\} d z d y d x, \delta_{n \rho}=\frac{1}{2 \pi L_{x} n^{2}} \int_{0}^{t} e_{n \rho}(\tau) \times \\
& \times \int_{0}^{L_{x} L_{y}}\left\{y s_{n}(y)+\frac{L_{y}}{2 \pi n}\left[c_{n}(y)-1\right]\right\} \int_{0}^{L_{z}}\left\{z s_{n}(z)+\frac{L_{z}}{2 \pi n}\left[c_{n}(z)-1\right]\right\} D_{\rho}(x, y, z, T) d z d y[1- \\
& \left.-c_{n}(2 x)\right] d x d \tau+\frac{1}{2 \pi L_{y} n^{2}} \int_{0}^{t} e_{n \rho}(\tau) \int_{0}^{L_{x}}\left\{L_{x}+x s_{n}(2 x)+\frac{L_{x}}{\pi n}\left[c_{n}(2 x)-1\right] \int_{0}^{L_{y}}\left[1-c_{n}(2 y)\right] \int_{0}^{L_{z}}\left\{L_{z}+\right.\right. \\
& \left.+z s_{n}(2 z)+\frac{L_{z}}{2 \pi n}\left[c_{n}(2 z)-1\right]\right\} D_{\rho}(x, y, z, T) d z d y d x d \tau+\frac{1}{2 \pi L_{z} n^{2}} \int_{0}^{t} e_{n \rho}(\tau) \int_{0}^{L_{x}}\left\{x s_{n}(2 x)+\right. \\
& \left.+L_{x}+\frac{L_{x}}{\pi n}\left[c_{n}(2 x)-1\right]\right\} \int_{0}^{L_{y}}\left\{L_{y}+y s_{n}(y)+\frac{L_{y}}{2 \pi n}\left[c_{n}(y)-1\right] \int_{0}^{L_{z}}\left[1-c_{n}(2 z)\right] D_{\rho}(x, y, z, T) d z \times\right. \\
& \times d y d x d \tau-\frac{L_{x}^{2} L_{y}^{2} L_{z}^{2}}{\pi^{5} n^{6}} e_{n \rho}(t), \chi_{n I V}=\int_{0}^{L_{x}}\left\{x s_{n}(x)+\frac{L_{x}}{\pi n}\left[c_{n}(x)-1\right]\right\} \int_{0}^{L_{y}}\left\{L_{y}+\frac{L_{y}}{2 \pi n}\left[c_{n}(2 y)-1\right]+\right. \\
& \left.+y s_{n}(2 y)\right\} \int_{0}^{L_{z}} k_{I, V}(x, y, z, T)\left\{L_{z}+z s_{n}(2 z)+\frac{L_{z}}{2 \pi n}\left[c_{n}(2 z)-1\right]\right\} d z d y d x e_{n I}(t) e_{n V}(t), \\
& \lambda_{n \rho}=\int_{0}^{L_{x}}\left\{x s_{n}(x)+\frac{L_{x}}{\pi n}\left[c_{n}(x)-1\right]\right\} \int_{0}^{L_{y}}\left\{y s_{n}(y)+\frac{L_{y}}{\pi n}\left[c_{n}(y)-1\right]\right\} \int_{0}^{L_{z}}\left\{z s_{n}(z)+\frac{L_{z}}{\pi n}\left[c_{n}(z)-1\right]\right\} \times \\
& \times f_{\rho}(x, y, z, T) d z d y d x, b_{4}=\gamma_{n V} \gamma_{n I}^{2}-\gamma_{n I} \chi_{n I}^{2}, b_{3}=2 \gamma_{n V} \gamma_{n I} \delta_{n I}-\delta_{n I} \chi_{n I}^{2}-\delta_{n V} \chi_{n I} \gamma_{n I}, \\
& A=\sqrt{8 y+b_{3}^{2}-4 b_{2}}, b_{2}=\gamma_{n V} \delta_{n I}^{2}+2 \lambda_{n I} \gamma_{n V} \gamma_{n I}-\delta_{n V} \chi_{n I} \delta_{n I}+\left(\lambda_{n V}-\lambda_{n I}\right) \chi_{n I}^{2}, b_{1}=2 \lambda_{n I} \times \\
& \times \gamma_{n V} \delta_{n I}-\delta_{n V} \chi_{n I} \lambda_{n I}, y=\sqrt[3]{\sqrt{q^{2}+p^{3}}-q}-\sqrt[3]{\sqrt{q^{2}+p^{3}}+q}-\frac{b_{3}}{3 b_{4}}, p=\frac{3 b_{2} b_{4}-b_{3}^{2}}{9 b_{4}^{2}}, \\
& q=\left(2 b_{3}^{3}-9 b_{2} b_{3}+27 b_{1} b_{4}^{2}\right) / 54 b_{4}^{3} .
\end{aligned}
$$

We determine solutions of the Eqs.(4a) as the following series

$$
\Phi_{\rho 0}(x, y, z, t)=\sum_{n=1}^{N} a_{n \Phi \rho} c_{n}(x) c_{n}(y) c_{n}(z) e_{n \rho}(t)
$$

Coefficients $a_{n \Phi \rho}$ are not yet known. Let us previously transform the Eqs.(6) to the following integro-differential form

$$
\begin{aligned}
& \frac{x y z}{L_{x} L_{y} L_{z}} \int_{L_{x} L_{y} L_{z}}^{x} \int_{I}^{z} \Phi_{I}(u, v, w, t) d w d v d u=\int_{0}^{t} \int_{L_{y} L_{z}}^{y} \int_{\Phi I}^{z} D_{\Phi}(x, v, w, T) \frac{\partial \Phi_{I}(x, v, w, \tau)}{\partial x} d w d v d \tau \times \\
& \times \frac{y z}{L_{y} L_{z}}+\frac{x z}{L_{x} L_{z}} \int_{0}^{t} \int_{L_{x} L_{z}}^{x} \int_{\Phi I}^{z} D_{\Phi}(u, y, w, T) \frac{\partial \Phi_{I}(u, y, w, \tau)}{\partial y} d w d u d \tau+\frac{x y}{L_{x} L_{y}} \int_{0}^{t} \int_{L_{x} L_{y}}^{x} \int_{\Phi}^{y} D_{\Phi I}(u, v, z, T) \times \\
& \times \frac{\partial \Phi_{I}(u, v, z, \tau)}{\partial z} d v d u d \tau+\frac{x y z}{L_{x} L_{y} L_{z}} \int_{L_{x} L_{y} L_{z}}^{x} \int_{I, I}^{z} k_{I}(u, v, w, T) I^{2}(u, v, w, \tau) d w d v d u-(6 a) \\
& -\frac{x y z}{L_{x} L_{y} L_{z}} \int_{L_{x} L_{y} L_{z}}^{x} \int_{I}^{z} k_{I}(u, v, w, T) I(u, v, w, \tau) d w d v d u+\frac{x y z}{L_{x} L_{y} L_{z}} \int_{L_{x} L_{y} L_{z}}^{x} \int_{\Phi I}^{z} f_{\Phi}(u, v, w) d w d v d u
\end{aligned}
$$




$$
\begin{aligned}
& \frac{x y z}{L_{x} L_{y} L_{z}} \int_{L_{x} L_{y} L_{z}}^{x} \int^{z} \Phi_{V}(u, v, w, t) d w d v d u=\int_{0}^{t} \int_{L_{y} L_{z}}^{y} \int_{\Phi V}^{z} D_{\Phi}(x, v, w, T) \frac{\partial \Phi_{V}(x, v, w, \tau)}{\partial x} d w d v d \tau \times \\
& \times \frac{y z}{L_{y} L_{z}}+\frac{x z}{L_{x} L_{z}} \int_{0}^{t} \int_{L_{x} L_{z}}^{z} D_{\Phi V}(u, y, w, T) \frac{\partial \Phi_{V}(u, y, w, \tau)}{\partial y} d w d u d \tau+\frac{x y}{L_{x} L_{y}} \int_{0}^{t} \int_{L_{x} L_{y}}^{x} \int_{\Phi V}^{y} D_{\Phi}(u, v, z, T) \times \\
& \times \frac{\partial \Phi_{V}(u, v, z, \tau)}{\partial z} d v d u d \tau+\frac{x y z}{L_{x} L_{y} L_{z}} \int_{L_{x} L_{y} L_{z}}^{x} \int_{V, V}^{z} k_{V}(u, v, w, T) V^{2}(u, v, w, \tau) d w d v d u- \\
& -\frac{x y z}{L_{x} L_{y} L_{z}} \int_{L_{x} L_{y} L_{z}}^{x} \int_{z}^{z} k_{V}(u, v, w, T) V(u, v, w, \tau) d w d v d u+\frac{x y z}{L_{x} L_{y} L_{z}} \int_{L_{x} L_{y}}^{x} \int_{L_{z}}^{y} f_{\Phi V}(u, v, w) d w d v d u .
\end{aligned}
$$

Substitution of the previously considered series in the Eqs.( $6 a$ ) leads to the following form

$$
\begin{aligned}
& -x y z \sum_{n=1}^{N} \frac{a_{n \Phi I}}{\pi^{3} n^{3}} s_{n}(x) s_{n}(y) s_{n}(z) e_{n I}(t)=-\frac{y z \pi}{L_{x} L_{y} L_{z}} \sum_{n=1}^{N} n a_{n \Phi I} s_{n}(x) e_{n I}(t) \int_{0}^{t} \int_{L_{y} L_{z}}^{y} \int_{n}^{z} c_{n}(v) c_{n}(w) \times \\
& \times D_{\Phi I}(x, v, w, T) d w d v d \tau-\frac{x z \pi}{L_{x} L_{y} L_{z}} \sum_{n=1}^{N} a_{n \Phi} \int_{0}^{t} \int_{L_{x} L_{z}}^{x} \int_{n}^{z} c_{n}(u) c_{n}(w) D_{\Phi I}(u, v, w, T) d w d u d \tau \times \\
& \times n s_{n}(y) e_{n \Phi I}(t)-\frac{x y \pi}{L_{x} L_{y} L_{z}} \sum_{n=1}^{N} n a_{n \Phi I} s_{n}(z) e_{n \Phi I}(t) \int_{0}^{t} \int_{L_{x} L_{y}}^{x} c_{n}(u) c_{n}(v) D_{\Phi I}(u, v, z, T) d v d u d \tau+ \\
& +\frac{x y z}{L_{x} L_{y} L_{z}} \int_{L_{x}}^{x} \int_{L_{y} L_{z}}^{y} \int_{I, I}^{z} k_{I, I}(u, v, w, T) I^{2}(u, v, w, \tau) d w d v d u+\int_{L_{x} L_{y} \int_{z}}^{y} \int_{L_{z}}^{z} f_{\Phi I}(u, v, w) d w d v d u \times \\
& \times \frac{x y z}{L_{x} L_{y} L_{z}}-\frac{x y z}{L_{x} L_{y} L_{z}} \int_{L_{x} L_{y} L_{z}}^{x} \int_{I}^{z} k_{I}(u, v, w, T) I(u, v, w, \tau) d w d v d u \\
& -x y z \sum_{n=1}^{N} \frac{a_{n \Phi V}}{\pi^{3} n^{3}} s_{n}(x) s_{n}(y) s_{n}(z) e_{n V}(t)=-\frac{y z \pi}{L_{x} L_{y} L_{z}} \sum_{n=1}^{N} n a_{n \Phi V} s_{n}(x) e_{n V}(t) \int_{0}^{t} \int_{L_{y} L_{z}}^{y} \int_{n}^{z} c_{n}(v) c_{n}(w) \times \\
& \times D_{\Phi V}(x, v, w, T) d w d v d \tau-\frac{x z \pi}{L_{x} L_{y} L_{z}} \sum_{n=1}^{N} n \iint_{0}^{t} \int_{L_{x}}^{x} \int_{L_{z}}^{z} c_{n}(u) c_{n}(w) D_{\Phi V}(u, v, w, T) d w d u d \tau \times \\
& \times a_{n \Phi V} s_{n}(y) e_{n \Phi V}(t)-\frac{x y \pi}{L_{x} L_{y} L_{z}} \sum_{n=1}^{N} n s_{n}(z) e_{n \Phi V}(t) \int_{0}^{t} \int_{0}^{x} \int_{x} L_{y}^{y} c_{n}(u) c_{n}(v) D_{\Phi V}(u, v, z, T) d v d u d \tau \times \\
& \times a_{n \Phi V}+\frac{x y z}{L_{x} L_{y} L_{z}} \int_{L_{x} L_{y} L_{z}}^{x} \int_{V, V}^{z} k_{V}(u, v, w, T) V^{2}(u, v, w, \tau) d w d v d u+\int_{L_{x} L_{y} L_{z}}^{x} \int_{\Phi V}^{z} f_{\Phi}(u, v, w) d w d v d u \times \\
& \times \frac{x y z}{L_{x} L_{y} L_{z}}-\frac{x y z}{L_{x} L_{y} L_{z}} \int_{L_{x} L_{y} L_{z}}^{x} \int_{V}^{z} k_{V}(u, v, w, T) V(u, v, w, \tau) d w d v d u .
\end{aligned}
$$

We determine coefficients $a_{n \Phi \rho}$ by using orthogonality condition on the scale of heterostructure. The condition gives us possibility to obtain relations to calculate $a_{n \Phi \rho}$ for any quantity $N$ of terms of considered series. In the common case equations for the required coefficients could be written as 
International Journal on Computational Science \& Applications (IJCSA) Vol.5, No.4, August 2015

$$
\begin{aligned}
& -\frac{L_{x}^{2} L_{y}^{2} L_{z}^{2}}{\pi^{5}} \sum_{n=1}^{N} \frac{a_{n \Phi I}}{n^{6}} e_{n \Phi I}(t)=-\frac{1}{2 \pi L_{x}} \sum_{n=1}^{N} \int_{0}^{t} \int_{0}^{L_{x}}\left[1-c_{n}(2 x)\right] \int_{0}^{L_{y}}\left\{L_{y}+y s_{n}(2 y)+\frac{L_{y}}{2 \pi n}\left[c_{n}(2 y)-1\right]\right\} \times \\
& \times \frac{a_{n \Phi I}}{n^{2}} \int_{0}^{L_{\tilde{z}}} D_{\Phi I}(x, y, z, T)\left\{z s_{n}(z)+\frac{L_{z}}{2 \pi n}\left[c_{n}(z)-1\right]\right\} d z d y d x e_{n \Phi I}(\tau) d \tau-\frac{1}{2 \pi} \sum_{n=1}^{N} \int_{0}^{t} \int_{0}^{L_{x}}\left\{x s_{n}(2 x)+\right. \\
& +L_{x}+\frac{L_{x}}{2 \pi n}\left[c_{n}(2 x)-1\right] \int_{0}^{L_{y}}\left[1-c_{n}(2 y)\right] \int_{0}^{L_{z}} D_{\Phi I}(x, y, z, T)\left\{z s_{n}(z)+\frac{L_{z}}{2 \pi n}\left[c_{n}(z)-1\right]\right\} d z d y d x \times \\
& \times a_{n \Phi I} \frac{e_{n \Phi I}(\tau)}{n^{2} L_{y}} d \tau-\frac{1}{2 \pi L_{x}} \sum_{n=1}^{N} \frac{a_{n \Phi I}}{n^{2}} \int_{0}^{t} \int_{0}^{L_{x}}\left\{x s_{n}(x)+\frac{L_{x}}{2 \pi n}\left[c_{n}(x)-1\right]\right\} \int_{0}^{L_{y}}\left\{y s_{n}(2 y)+\frac{L_{y}}{2 \pi n}\left[c_{n}(2 y)-1\right]+\right. \\
& \left.+L_{y}\right\}_{0}^{L_{z}}\left[1-c_{n}(2 y)\right] D_{\Phi I}(x, y, z, T) d z d y d x e_{n \Phi I}(\tau) d \tau+\frac{1}{\pi^{3}} \sum_{n=1}^{N} \frac{a_{n \Phi I}}{n^{3}} \int_{0}^{t} e_{n \Phi I}(\tau) \int_{0}^{L_{x}}\left\{\frac{L_{x}}{2 \pi n}\left[c_{n}(x)-1\right]+\right. \\
& \left.+x s_{n}(x)\right\} \int_{0}^{L_{y}}\left\{y s_{n}(y)+\frac{L_{y}}{2 \pi n}\left[c_{n}(y)-1\right]\right\} \int_{0}^{L_{z}} I^{2}(x, y, z, t) k_{I, I}(x, y, z, T)\left\{\frac{L_{z}}{2 \pi n}\left[c_{n}(z)-1\right]+\right. \\
& \left.+z s_{n}(z)\right\} d z d y d x-\frac{1}{\pi^{3}} \sum_{n=1}^{N} \frac{a_{n \Phi I}}{n^{3}} \int_{0}^{t} e_{n \Phi I}(\tau) \int_{0}^{L_{x}}\left\{x s_{n}(x)+\frac{L_{x}}{2 \pi n}\left[c_{n}(x)-1\right]\right\} \int_{0}^{L_{y}}\left\{\frac{L_{y}}{2 \pi n}\left[c_{n}(y)-1\right]+\right. \\
& \left.+y s_{n}(y)\right\} \int_{0}^{L_{z}}\left\{z s_{n}(z)+\frac{L_{z}}{2 \pi n}\left[c_{n}(z)-1\right]\right\} k_{I}(x, y, z, T) I(x, y, z, t) d z d y d x+\frac{1}{\pi^{3}} \sum_{n=1}^{N} \frac{a_{n \Phi I}}{n^{3}} \times \\
& \times \int_{0}^{t} e_{n \Phi I}(\tau) \int_{0}^{L_{x}}\left\{x s_{n}(x)+\frac{L_{x}}{2 \pi n}\left[c_{n}(x)-1\right]\right\} \int_{0}^{L_{y}}\left\{y s_{n}(y)+\frac{L_{y}}{2 \pi n}\left[c_{n}(y)-1\right]\right\} \int_{0}^{L_{z}}\left\{\frac{L_{z}}{2 \pi n}\left[c_{n}(z)-1\right]+\right. \\
& \left.+z s_{n}(z)\right\} f_{\Phi I}(x, y, z) d z d y d x \\
& -\frac{L_{x}^{2} L_{y}^{2} L_{z}^{2}}{\pi^{5}} \sum_{n=1}^{N} \frac{a_{n \Phi V}}{n^{6}} e_{n \Phi V}(t)=-\frac{1}{2 \pi L_{x}} \sum_{n=1}^{N} \int_{0}^{t} \int_{0}^{L_{x}}\left[1-c_{n}(2 x)\right] \int_{0}^{L_{y}}\left\{L_{y}+y s_{n}(2 y)+\frac{L_{y}}{2 \pi n}\left[c_{n}(2 y)-1\right]\right\} \times \\
& \times \frac{a_{n \Phi V}}{n^{2}} \int_{0}^{L_{z}} D_{\Phi V}(x, y, z, T)\left\{z s_{n}(z)+\frac{L_{z}}{2 \pi n}\left[c_{n}(z)-1\right]\right\} d z d y d x e_{n \Phi V}(\tau) d \tau-\frac{1}{2 \pi} \sum_{n=1}^{N} \int_{0}^{t} \int_{0}^{L_{x}}\left\{x s_{n}(2 x)+\right. \\
& +L_{x}+\frac{L_{x}}{2 \pi n}\left[c_{n}(2 x)-1\right] \int_{0}^{L_{y}}\left[1-c_{n}(2 y)\right] \int_{0}^{L_{z}} D_{\Phi V}(x, y, z, T)\left\{z s_{n}(z)+\frac{L_{z}}{2 \pi n}\left[c_{n}(z)-1\right]\right\} d z d y d x \times \\
& \times a_{n \Phi V} \frac{e_{n \Phi V}(\tau)}{n^{2} L_{y}} d \tau-\frac{1}{2 \pi L_{x}} \sum_{n=1}^{N} \frac{a_{n \Phi V}}{n^{2}} \int_{0}^{t L_{x}} \int_{0}\left\{x s_{n}(x)+\frac{L_{x}}{2 \pi n}\left[c_{n}(x)-1\right]\right\} \int_{0}^{L_{y}}\left\{y s_{n}(2 y)+\frac{L_{y}}{2 \pi n}\left[c_{n}(2 y)-1\right]+\right. \\
& \left.+L_{y}\right\} \int_{0}^{L_{z}}\left[1-c_{n}(2 y)\right] D_{\Phi V}(x, y, z, T) d z d y d x e_{n \Phi V}(\tau) d \tau+\frac{1}{\pi^{3}} \sum_{n=1}^{N} \frac{a_{n \Phi \Phi}}{n^{3}} \int_{0}^{t} e_{n \Phi V}(\tau) \int_{0}^{L_{x}}\left\{\frac{L_{x}}{2 \pi n}\left[c_{n}(x)-1\right]+\right. \\
& \left.+x s_{n}(x)\right\} \int_{0}^{L_{y}}\left\{y s_{n}(y)+\frac{L_{y}}{2 \pi n}\left[c_{n}(y)-1\right]\right\} \int_{0}^{L_{z}} V^{2}(x, y, z, t) k_{V, V}(x, y, z, T)\left\{\frac{L_{z}}{2 \pi n}\left[c_{n}(z)-1\right]+\right. \\
& \left.+z s_{n}(z)\right\} d z d y d x-\frac{1}{\pi^{3}} \sum_{n=1}^{N} \frac{a_{n \Phi V}}{n^{3}} \int_{0}^{t} e_{n \Phi V}(\tau) \int_{0}^{L_{x}}\left\{x s_{n}(x)+\frac{L_{x}}{2 \pi n}\left[c_{n}(x)-1\right] \int_{0}^{L_{y}}\left\{\frac{L_{y}}{2 \pi n}\left[c_{n}(y)-1\right]+\right.\right.
\end{aligned}
$$




$$
\begin{gathered}
\left.+y s_{n}(y)\right\} \int_{0}^{L_{z}}\left\{z s_{n}(z)+\frac{L_{z}}{2 \pi n}\left[c_{n}(z)-1\right]\right\} k_{V}(x, y, z, T) V(x, y, z, t) d z d y d x+\frac{1}{\pi^{3}} \sum_{n=1}^{N} \frac{a_{n \Phi V}}{n^{3}} \times \\
\times \int_{0}^{t} e_{n \Phi V}(\tau) \int_{0}^{L_{x}}\left\{x s_{n}(x)+\frac{L_{x}}{2 \pi n}\left[c_{n}(x)-1\right]\right\} \int_{0}^{L_{y}}\left\{y s_{n}(y)+\frac{L_{y}}{2 \pi n}\left[c_{n}(y)-1\right]\right\} \int_{0}^{L_{z}}\left\{\frac{L_{z}}{2 \pi n}\left[c_{n}(z)-1\right]+\right. \\
\left.+z s_{n}(z)\right\} f_{\Phi V}(x, y, z) d z d y d x .
\end{gathered}
$$

It should be noted, that we consider analytical algorithm of calculation of concentrations of dopant and radiation defects. We use only two simplifications: $(i)$ we consider approximate using of Arrhenius law for analytical calculation of appropriate integrals; (ii) we consider so approximations of initial distributions, which give a possibility to calculate appropriate integrals analytically. Summation of considered series after calculation of integrals could be done by using any soft, which gives a possibility of the summation.

\section{Discussion}

In this section we used calculated in the previous sections relations for analysis the spatio-temporal distributions of concentrations of infused and implanted dopants. During analysis of spatial distributions of concentrations of dopants it has been shown, that inhomogeneity of heterostructure gives us possibility to increase sharpness of $p$ - $n$-heterojunctions both single and framework transistors. Figs. 2 show spatial distributions of concentrations of dopants in direction, which is perpendicular to interface between epitaxial layer substrate. Increasing of number of curve corresponds to increasing of difference between values of dopant diffusion coefficient in layers of heterostructure under condition, when value of dopant diffusion coefficient in epitaxial layer is larger, than value of dopant diffusion coefficient in substrate. We calculate distributions of concentrations of dopants for larger value of dopant diffusion coefficient in doped area in comparison with undoped one.

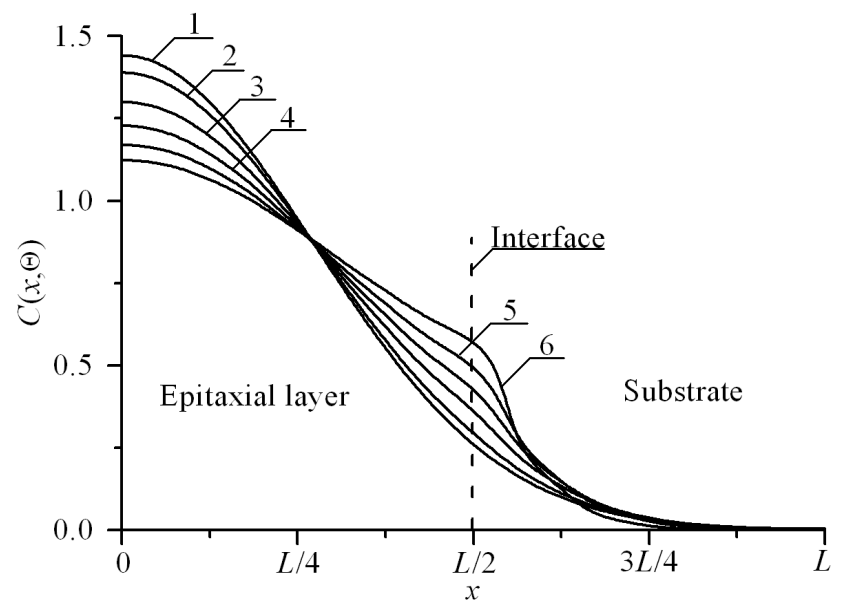

Fig.2a. Distributions of concentration of infused dopant in heterostructure from Figs. 1 and 2 


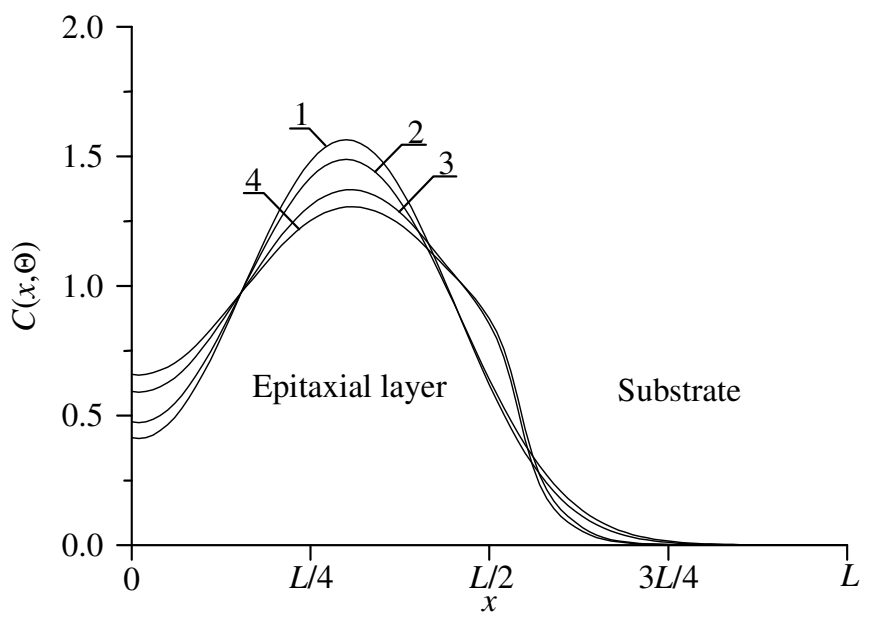

Fig. $2 b$. Distributions of concentration of implanted dopant. Curves 1 and 3 corresponds to annealing time $\Theta$ $=0.0048\left(L_{x}{ }^{2}+L_{y}{ }^{2}+L_{z}{ }^{2}\right) / D_{0}$. Curves 2 and 4 corresponds to annealing time $\Theta=0.0057\left(L_{x}{ }^{2}+L_{y}{ }^{2}+L_{z}{ }^{2}\right) / D_{0}$

One can find from the Figs.2 show, that in homogeneity of heterostructure leads to increasing sharpness of $p-n$-junctions and homogeneity of distribution of concentration of dopant. However in this situation one shall optimize annealing of dopant and/or radiation defects during manufacturing $p$ - $n$-junctions and transistors. Reasons of this necessity is following. If annealing time of infused dopant is small, dopant have no enough time to achieve interface between layers of heterostructure. In this situation one can not find any changing of distribution of concentration of dopant with presents of interface between layers of hterostructure. If annealing time is too large, distribution of concentration of dopant became too homogenous. We determine optimal value of annealing time framework recently introduced criterion [17,23-31]. To use the criterion one shall approximate real distribution of concentration of dopant by step-wise function $\psi(x, y, z)$. After that we determine required optimal value of annealing time by minimization the following meansquared error

$$
U=\frac{1}{L_{x} L_{y} L_{z}} \int_{0}^{L_{L} L_{y}} \int_{0}^{L_{z}} \int_{0}[C(x, y, z, \Theta)-\psi(x, y, z)] d z d y d x
$$

In this case we obtain optimal value of annealing time, which corresponds to minimal difference between real distribution of concentration of dopant and required step-wise approximation of the concentration.

Dependences of optimal values of annealing time of dopant are presented on Figs. 3. Curve 1 is the dependence of dimensionless optimal annealing time on the relation $a / L$ and $\xi=\gamma=0$ for equal to each other values of dopant diffusion coefficient in all parts of heterostructure. Curve 2 is the dependence of dimensionless optimal annealing time on value of parameter $\varepsilon$ for $a / L=1 / 2$ and $\xi=\gamma$ $=0$. Curve 3 is the dependence of dimensionless optimal annealing time on value of parameter $\xi$ for $a / L=1 / 2$ and $\varepsilon=\gamma=0$. Curve 4 is the dependence of dimensionless optimal annealing time on value of parameter $\gamma$ for $a / L=1 / 2$ and $\varepsilon=\xi=0$. It is known, that radiation defects after ion implantation should be annealed. In the ideal case annealing time of radiation defects should be so, that dopant during the annealing should be achieves interface between layers of heterostructure. If the dopant has not enough time to achieve the interface during the annealing, it is practicably to use additional annealing of dopant. Dependences of optimal values of the above additional annealing 
of dopant are presented on the Fig. $3 b$. Necessity of annealing of radiation defects leads to smaller values of optimal annealing time of implanted dopant in comparison with the optimal annealing time of infused dopant. It is known, that diffusion type of doping did not leads to any radiation damage of materials of heterostructure. However ion implantation gives us possibility gives us possibility to decrease mismatch-induced stress in heterostructure [32].

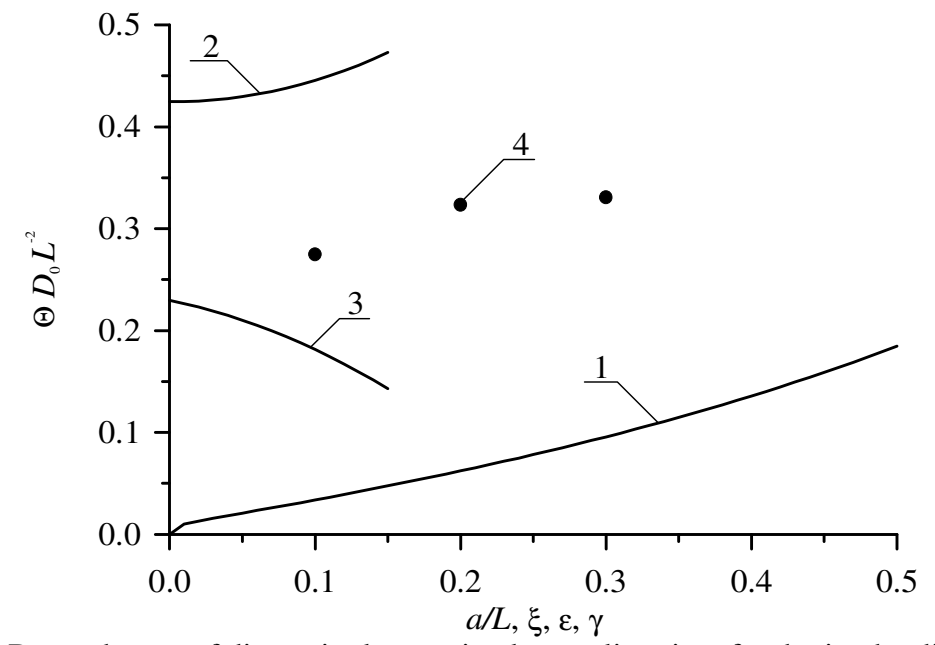

Fig.3a. Dependences of dimensionless optimal annealing time for doping by diffusion

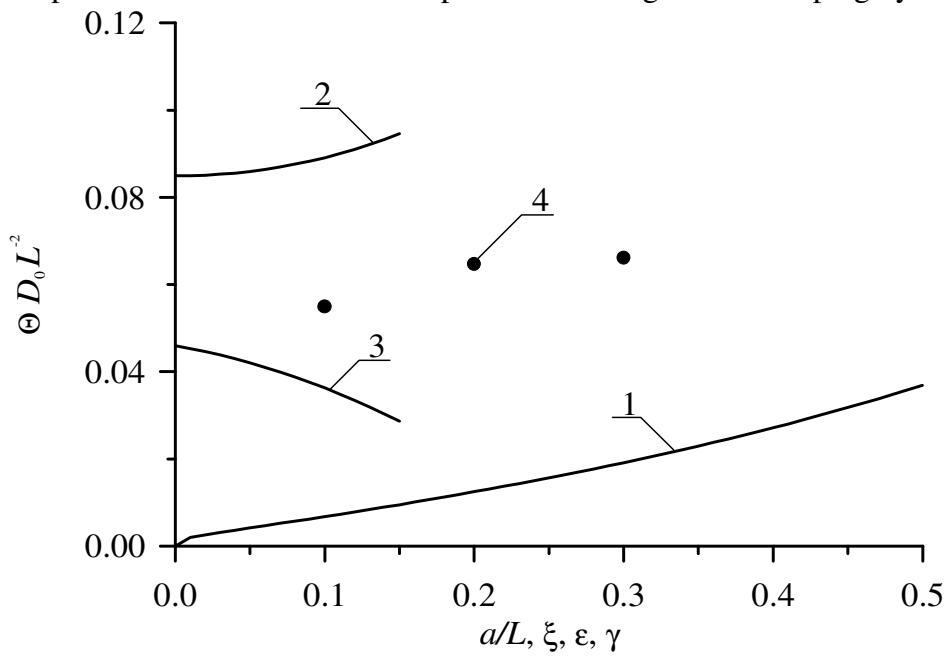

Fig.3b. Dependences of dimensionless optimal annealing time for doping by ion implantation

\section{CONCLUSIONS}

In this paper we introduce an approach to manufacture more compact logical elements based on bipolar transistor. The approach based on fabrication a heterostructure with required configuration, doping by diffusion or ion implantation of required parts of heterostructure and optimal annealing of dopant and/or radiation defects. At the same time we consider an analytical approach to model technological processes, which gives us possibility to do without crosslinking solutions at the interfaces between the layers of the heterostructure. 


\section{ACKNOWLEDGEMENTS}

This work is supported by the agreement of August 27, 2013 № 02.B.49.21.0003 between The Ministry of education and science of the Russian Federation and Lobachevsky State University of Nizhni Novgorod and educational fellowship for scientific research of Government of Russian and of Nizhny Novgorod State University of Architecture and Civil Engineering.

\section{REFERENCES}

[1] V.I. Lachin, N.S. Savelov. Electronics (Phoenix, Rostov-na-Donu, 2001).

[2] A.G. Alexenko, I.I. Shagurin. Microcircuitry (Radio and communication, Moscow, 1990).

[3] N.A. Avaev, Yu.E. Naumov, V.T. Frolkin. Basis of microelectronics (Radio and communication, Moscow, 1991).

[4] D. Fathi, B. Forouzandeh. Accurate analysis of global interconnects in nano-FpGAs. Nano. Vol. 4 (3). P. 171-176 (2009).

[5] D. Fathi, B. Forouzandeh, N. Masoumi. New enhanced noise analysis in active mixers in nanoscale technologies. Nano. Vol. 4 (4). P. 233-238 (2009).

[6] S.A. Chachuli, P.N.A. Fasyar, N. Soin, N.M. Karim, N. Yusop. Pareto ANOVA analysis for CMOS $0.18 \mu \mathrm{m}$ two-stage Op-amp. Mat. Sci. Sem. Proc. Vol. 24. P. 9-14 (2014).

[7] A.O. Ageev, A.E. Belyaev, N.S. Boltovets, V.N. Ivanov, R.V. Konakova, Ya.Ya. Kudrik, P.M. Litvin, V.V. Milenin, A.V. Sachenko. Influence of displacement of the electron-hole equilibrium on the process of transition metals diffusion in GaAs. Semiconductors. Vol. 43 (7). P. 897-903 (2009).

[8] Jung-Hui Tsai, Shao-Yen Chiu, Wen-Shiung Lour, Der-Feng Guo. High-performance InGaP/GaAs pnp $\delta$-doped heterojunction bipolar transistor. Semiconductors. Vol. 43 (7). P. 971-974 (2009).

[9] O.V. Alexandrov, A.O. Zakhar'in, N.A. Sobolev, E.I. Shek, M.M. Makoviychuk, E.O. Parshin. Formation of donor centers after annealing of dysprosium and holmium implanted silicon. Semiconductors. Vol. 32 (9). P. 1029-1032 (1998).

[10] M.J. Kumar, T.V. Singh. Quantum confinement effects in strained silicon MOSFETs. Int. J. Nanoscience. Vol. 7 (2-3). P. 81-84 (2008).

[11] P. Sinsermsuksakul, K. Hartman, S.B. Kim, J. Heo, L. Sun, H.H. Park, R. Chakraborty, T. Buonassisi, R.G. Gordon. Enhancing the efficiency of $\mathrm{SnS}$ solar cells via band-offset engineering with a zinc oxysulfide buffer layer. Appl. Phys. Lett. Vol. 102 (5). P. $053901-053905$ (2013).

[12] J.G. Reynolds, C.L. Reynolds, Jr.A. Mohanta, J.F. Muth, J.E. Rowe, H.O. Everitt, D.E. Aspnes. Shallow acceptor complexes in p-type ZnO. Appl. Phys. Lett. Vol. 102 (15). P. 152114-152118 (2013).

[13] K.K. Ong, K.L. Pey, P.S. Lee, A.T.S. Wee, X.C. Wang, Y.F. Chong. Dopant distribution in the recrystallization transient at the maximum melt depth induced by laser annealing. Appl. Phys. Lett. 89 (17), 172111-172114 (2006).

[14] H.T. Wang, L.S. Tan, E. F. Chor. Pulsed laser annealing of Be-implanted GaN. J. Appl. Phys. 98 (9), 094901-094905 (2006).

[15] S.T. Shishiyanu, T.S. Shishiyanu, S.K. Railyan. Shallow p-n-junctions in Si prepared by pulse photon annealing. Semiconductors. Vol.36 (5). P. 611-617 (2002).

[16] Yu.V. Bykov, A.G. Yeremeev, N.A. Zharova, I.V. Plotnikov, K.I. Rybakov, M.N. Drozdov, Yu.N. Drozdov, V.D. Skupov. Diffusion processes in semiconductor structures during microwave annealing. Radiophysics and Quantum Electronics. Vol. 43 (3). P. 836-843 (2003).

[17] E.L. Pankratov, E.A. Bulaeva. Doping of materials during manufacture p-n-junctions and bipolar transistors. Analytical approaches to model technological approaches and ways of optimization of distributions of dopants. Reviews in Theoretical Science. Vol. 1 (1). P. 58-82 (2013).

[18] V.V. Kozlivsky. Modification of semiconductors by proton beams (Nauka, Sant-Peterburg, 2003, in Russian).

[19] Z.Yu. Gotra. Technology of microelectronic devices (Radio and communication, Moscow, 1991).

[20] V.L. Vinetskiy, G.A. Kholodar', Radiative physics of semiconductors. ("Naukova Dumka", Kiev, 1979, in Russian).

[21] P.M. Fahey, P.B. Griffin, J.D. Plummer. Point defects and dopant diffusion in sikicon. Rev. Mod. Phys. 1989. Vol. 61. № 2. P. 289-388.

[22] M.L. Krasnov, A.I. Kiselev, G.I. Makarenko. Integral equations ("Science", Moscow, 1976). 
International Journal on Computational Science \& Applications (IJCSA) Vol.5, No.4, August 2015

[23] E.L. Pankratov. Dopant diffusion dynamics and optimal diffusion time as influenced by diffusioncoefficient nonuniformity. Russian Microelectronics. 2007. V.36 (1). P. 33-39.

[24] E.L. Pankratov. Redistribution of dopant during annealing of radiative defects in a multilayer structure by laser scans for production an implanted-junction rectifiers. Int. J. Nanoscience. Vol. 7 (4-5). P. 187-197 (2008).

[25] E.L. Pankratov. Decreasing of depth of implanted-junction rectifier in semiconductor heterostructure by optimized laser annealing. J. Comp. Theor. Nanoscience. Vol. 7 (1). P. 289-295 (2010).

[26] E.L. Pankratov, E.A. Bulaeva. Application of native inhomogeneities to increase compactness of vertical field-effect transistors. J. Comp. Theor. Nanoscience. Vol. 10 (4). P. 888-893 (2013).

[27] E.L. Pankratov, E.A. Bulaeva. Optimization of doping of heterostructure during manufacturing of p-in-diodes. Nanoscience and Nanoengineering. Vol. 1 (1). P. 7-14 (2013).

[28] E.L. Pankratov, E.A. Bulaeva. An approach to decrease dimensions of field-effect transistors. Universal Journal of Materials Science. Vol. 1 (1). P.6-11 (2013).

[29] E.L. Pankratov, E.A. Bulaeva. An approach to manufacture a heterobipolar transistors in thin film structures. On the method of optimization. Int. J. Micro-Nano Scale Transp. Vol. 4 (1). P. 17-31 (2014).

[30] E.L. Pankratov, E.A. Bulaeva. Application of native inhomogeneities to increase compactness of vertical field-effect transistors. J. Nanoengineering and Nanomanufacturing. Vol. 2 (3). P. 275-280 (2012).

[31] E.L. Pankratov, E.A. Bulaeva. Influence of drain of dopant on distribution of dopant in diffusionheterojunction rectifiers. J. Adv. Phys. Vol. 2 (2). P. 147-150 (2013).

[32] E.L. Pankratov, E.A. Bulaeva. Decreasing of mechanical stress in a semiconductor heterostructure by radiation processing. J. Comp. Theor. Nanoscience. Vol. 11 (1). P. 91-101 (2014).

\section{Authors}

Pankratov Evgeny Leonidovich was born at 1977. From 1985 to 1995 he was educated in a secondary school in Nizhny Novgorod. From 1995 to 2004 he was educated in Nizhny Novgorod State University: from 1995 to 1999 it was bachelor course in Radiophysics, from 1999 to 2001 it was master course in Radiophysics with specialization in Statistical Radiophysics, from 2001 to 2004 it was PhD course in Radiophysics. From 2004 to 2008 E.L. Pankratov was a leading technologist in Institute for Physics of Microstructures. From 2008 to 2012 E.L. Pankratov was a senior lecture/Associate Professor of Nizhny Novgorod State University of Architecture and Civil Engineering. Now E.L. Pankratov is in his Full Doctor course in Radiophysical Department of Nizhny Novgorod State University. He has 105 published papers in area of his researches.

Bulaeva Elena Alexeevna was born at 1991. From 1997 to 2007 she was educated in secondary school of village Kochunovo of Nizhny Novgorod region. From 2007 to 2009 she was educated in boarding school "Center for gifted children". From 2009 she is a student of Nizhny Novgorod State University of Architecture and Civil Engineering (spatiality "Assessment and management of real estate"). At the same time she is a student of courses "Translator in the field of professional communication" and "Design (interior art)" in the University. E.A. Bulaeva was a contributor of grant of President of Russia (grant № MK-548.2010.2). She has 52 published papers in area of her researches. 